\title{
Epigenetic hypomethylation and upregulation of matrix metalloproteinase 9 in Kawasaki disease
}

\author{
Ho-Chang Kuo ${ }^{1,2}$, Sung-Chou Liं ${ }^{3}$, Lien-Hung Huang ${ }^{3}$ and Ying-Hsien Huang ${ }^{1,2,4}$ \\ ${ }^{1}$ Department of Pediatrics, Kaohsiung Chang Gung Memorial Hospital, Chang Gung University College of Medicine, Kaohsiung, \\ Taiwan \\ ${ }^{2}$ Kawasaki Disease Center, Kaohsiung Chang Gung Memorial Hospital, Kaohsiung, Taiwan \\ ${ }^{3}$ Department of Medical Research, Genomics and Proteomics Core Laboratory, Kaohsiung Chang Gung Memorial Hospital, \\ Chang Gung University College of Medicine, Kaohsiung, Taiwan \\ ${ }^{4}$ Department of Pediatrics, Chiayi Chang Gung Memorial Hospital, Kaohsiung, Taiwan \\ Correspondence to: Ying-Hsien Huang, email: yhhuang123@yahoo.com.tw \\ Keywords: matrix metalloproteinase, genetic methylation, Kawasaki disease, Immunology and Microbiology Section, Immune \\ response, Immunity \\ Received: April 05, $2017 \quad$ Accepted: June 28, $2017 \quad$ Published: July 28, 2017
}

Copyright: Kuo et al. This is an open-access article distributed under the terms of the Creative Commons Attribution License 3.0 (CC BY 3.0), which permits unrestricted use, distribution, and reproduction in any medium, provided the original author and source are credited.

\section{ABSTRACT}

Background: Kawasaki disease (KD) is a type of febrile coronary vasculitis occurring in children. Some researchers have suggested that changes in genetic signatures, such as matrix metalloproteinases (MMPs), are critical markers for cardiovascular diseases. This study aims to provide a comprehensive survey of global DNA methylation levels and MMP transcripts of KD patients compared to control subjects.

Materials and Methods: For chips studies, we recruited a total of $18 \mathrm{KD}$ patients, prior to receiving intravenous immunoglobulin (IVIG) and at least 3 weeks after IVIG treatment, as well as 18 healthy and 18 febrile control subjects. We applied Illumina HumanMethylation450 BeadChip and Affymetrix GeneChip ${ }^{\circledR}$ Human Transcriptome Array 2.0 to evaluate their CpG markers and expression levels, respectively. Then we used a separate cohort to carry out real-time quantitative PCR validations of mRNA levels.

Results: The expressions of mRNA levels of MMP-8, -9, and -25 were significantly upregulated in KD patients compared to the healthy and febrile controls. Once KD patients underwent IVIG treatment, these MMPs considerably decreased. In particular, the methylation status of CpG sites of MMP-9 indicated a significant opposite tendency between both stages of not only the KD samples but also the controls. We also observed the mRNA level of MMP-9 to be higher in KD patients with coronary arterial lesion formation.

Conclusion: This study is the first to report epigenetic hypomethylation, an increased MMP-9 transcript, and the upregulation of MMP-9 in KD patients who had formed coronary arterial lesions.

\section{INTRODUCTION}

Kawasaki disease (KD), also known as mucocutaneous lymph node syndrome or infantile periarteritis nodosa, inflames the walls of both smalland medium-sized blood vessels (vasculitis), particularly coronary arteries, throughout the body. KD typically affects children under the age of five years [1]. The most serious cardiovascular complications of KD are the result of coronary artery lesions (CALs) caused by inflammation, including myocardial infarctions, coronary artery fistula formations [2], coronary artery dilatations, and coronary artery aneurysms (CAAs) [3]. Although this disease can be controlled, nearly $20 \%$ of children who do not receive treatment suffer a CAA [2], which may lead to death in severe cases. 
Matrix metalloproteinases (MMPs) may maintain the structure and function of coronary vascular walls [4]. MMP-9 and -7 are well known to influence CAA formation in $\mathrm{KD}[5,6]$. Shimizu et al. reported that haplotype analyses showed MMP-3 and -12 in the gene cluster on Chromosome 11 in CAA formation in $\mathrm{KD}$ patients [7]. The proinflammatory cytokine tumor necrosis factor- $\alpha$ (TNF- $\alpha$ ) that stimulates MMP-9 production was also correlated with vascular damage in a KD model [8].

Epigenetics describes the acetylation pattern of the genome and DNA methylation and subsequently leads to changes in the chromatin structure [9]. In short, DNA methylation can deactivate genes through DNA methyltransferases [10]. In contrast, demethylation alterations of $\mathrm{CpG}$ sites indicate a contradictory change in gene expression [11]. In previous studies, we found that IVIG treatment significantly altered methylation patterns in KD patients [12] and that KD patients demonstrated considerably increased mRNA expression in TLRs and hypomethylation at the gene promoters of TLRs [13]. IVIG treatment can restore the methylation level of TLRs and decrease these TLRs' mRNA expression [13]. No studies have yet surveyed all the MMP-1 28 in the same report or performed analysis with methylation profiles. Therefore, in this study, we comprehensively examined the mRNA expressions of MMPs-1 28 and analyzed changes in methylation levels in two different stages of KD patients, as well as two types of controls.

\section{RESULTS}

\section{Differential expression of MMP mRNA levels among KD patients and controls and changes after IVIG treatment}

To investigate MMP1-28 transcripts expression, we used Affymetrix GeneChip ${ }^{\circledR}$ Human Transcriptome Array 2.0 to determine their expression levels. Table 1 shows that KD patients had a differential expression of MMPs when compared to both the healthy and febrile control subjects. The mRNA levels of MMP-8, -9 , and -25 were significantly higher in KD patients than in the healthy control and febrile control groups. These values significantly decreased in KD patients after undergoing IVIG treatment (Figure 1). We observed no noteworthy differences in the remaining MMPs among the groups or in KD patients following IVIG treatment.

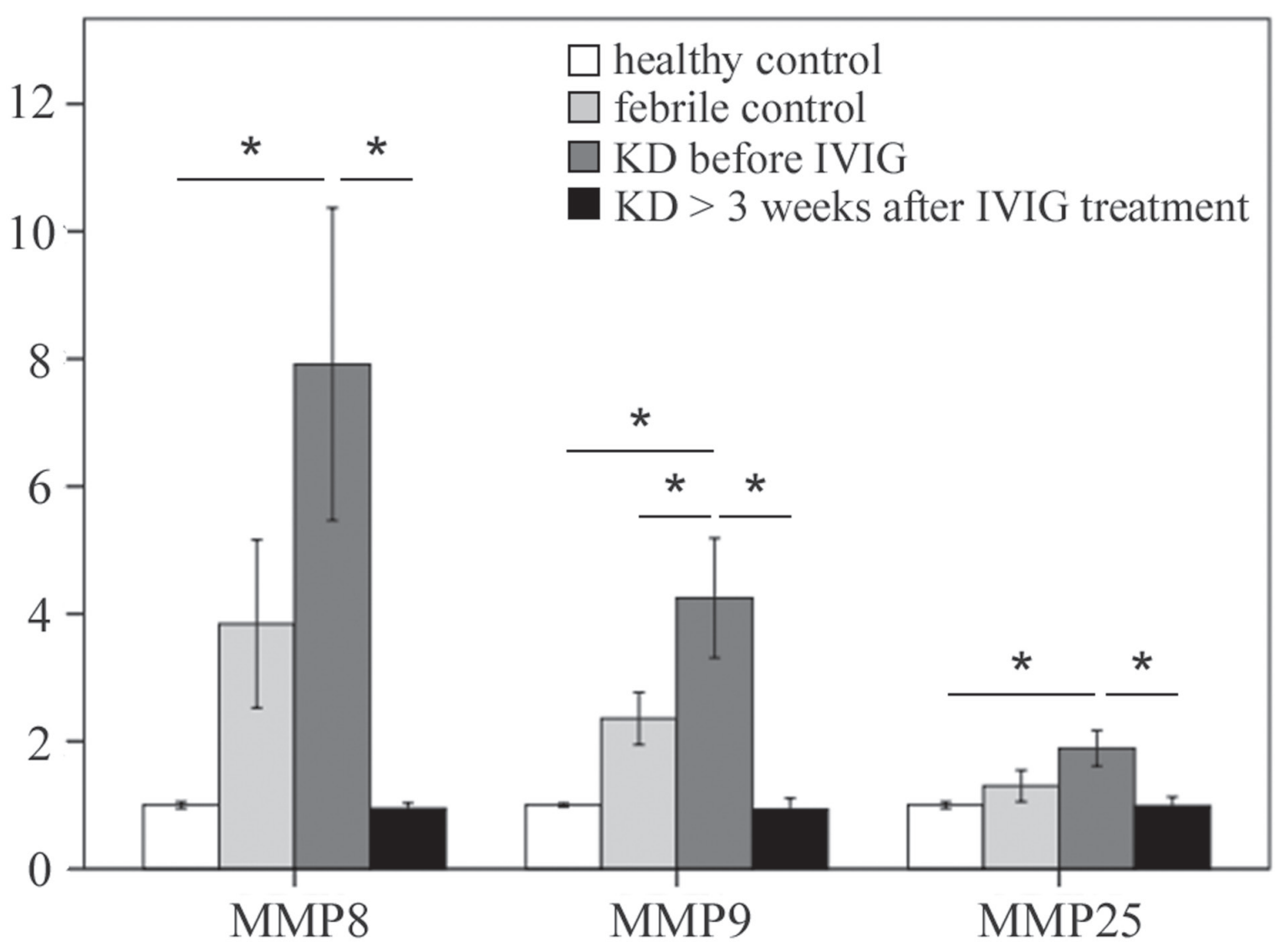

Figure 1: Comparison of matrix metalloproteinase (MMP) -8, -9, and -25 mRNA expressions by GeneChip ${ }^{\circledR}$ Human Transcriptome Array 2.0 between acute-stage Kawasaki disease patients and control subjects. * indicates significance $(p$ $<0.05)$. Data are expressed as mean \pm standard error for the three replications. 
Table 1: Transcripts expressions of matrix metalloproteinases (MMPs) between Kawasaki disease patients and control subjects

\begin{tabular}{|c|c|c|c|c|c|c|c|}
\hline Symbol & RefSeq & Fold-Change & $p$ value & Fold-Change & $p$ value & Fold-Change & $p$ value \\
\hline & & (KD1 vs. HC) & (KD1 vs. HC) & (KD1 vs. FC) & (KD1 vs. FC) & (KD3 vs. KD1) & (KD3 vs. KD1) \\
\hline MMP1 & NM_001145938 & 1.067 & 0.267 & -1.046 & 0.425 & -1.053 & 0.366 \\
\hline MMP2 & NM_001127891 & -1.004 & 0.970 & -1.005 & 0.960 & 1.092 & 0.363 \\
\hline MMP3 & NM_002422 & 1.009 & 0.865 & -1.085 & 0.163 & 1.014 & 0.803 \\
\hline MMP7 & NM_002423 & 1.011 & 0.876 & -1.038 & 0.582 & -1.001 & 0.990 \\
\hline MMP8 & NM_002424 & 7.143 & $0.001 *$ & 2.176 & 0.083 & -7.587 & $0.001 *$ \\
\hline MMP9 & NM_004994 & 4.062 & $0.000 *$ & 1.771 & $0.037 *$ & -4.481 & $0.000 *$ \\
\hline MMP10 & NM_002425 & -1.008 & 0.888 & -1.104 & 0.093 & 1.020 & 0.709 \\
\hline MMP11 & NM_005940 & -1.024 & 0.825 & -1.042 & 0.709 & 1.097 & 0.407 \\
\hline MMP12 & NM_002426 & 1.050 & 0.389 & -1.046 & 0.427 & -1.013 & 0.814 \\
\hline MMP13 & NM_002427 & 1.008 & 0.859 & -1.100 & 0.060 & 1.029 & 0.531 \\
\hline MMP14 & NM_004995 & 1.072 & 0.449 & -1.059 & 0.531 & 1.029 & 0.756 \\
\hline MMP15 & NM_002428 & -1.023 & 0.817 & -1.056 & 0.588 & 1.064 & 0.537 \\
\hline MMP16 & NM_005941 & 1.007 & 0.928 & -1.115 & 0.171 & 1.049 & 0.528 \\
\hline MMP17 & NM_016155 & -1.024 & 0.797 & -1.086 & 0.388 & 1.035 & 0.717 \\
\hline MMP19 & NM_002429 & 1.055 & 0.580 & -1.017 & 0.864 & -1.001 & 0.988 \\
\hline MMP20 & NM_004771 & -1.014 & 0.789 & -1.093 & 0.111 & 1.026 & 0.620 \\
\hline MMP21 & NM_147191 & -1.036 & 0.546 & -1.034 & 0.567 & 1.059 & 0.336 \\
\hline MMP23B & NM_006983 & -1.060 & 0.580 & -1.070 & 0.527 & 1.084 & 0.449 \\
\hline MMP24 & NM_006690 & -1.014 & 0.904 & -1.027 & 0.822 & 1.052 & 0.669 \\
\hline MMP25 & NM_022468 & 1.859 & $0.013 *$ & 1.473 & 0.082 & -1.915 & $0.010 *$ \\
\hline MMP26 & NM_021801 & -1.030 & 0.619 & -1.093 & 0.157 & 1.069 & 0.272 \\
\hline MMP27 & NM_022122 & 1.000 & 0.999 & -1.047 & 0.438 & 1.055 & 0.369 \\
\hline MMP28 & NM_001032278 & 1.011 & 0.914 & -1.015 & 0.877 & 1.029 & 0.767 \\
\hline
\end{tabular}

KD1: Kawasaki disease before IVIG treatment; KD3: Kawasaki disease > 3 weeks after IVIG treatment; FC: febrile control; $\mathrm{HC}$ : healthy control.

\section{Significantly altered CpG sites on MMPs between KD patients and controls}

We adopted Illumina HumanMethylation450 BeadChip (Illumina) to assess the methylation patterns of CpG sites on MMPs between KD patients and both types of control subjects (Table 2a-2g). The MMP methylation levels were found to vary considerably in the acute stage of KD patients compared to the healthy and febrile controls (Table 2a-2g). Furthermore, the methylation levels of MMP1-28 were significantly lower in acutestage KD patients compared to the healthy and febrile controls (Table 2a-2g). After KD patients underwent IVIG treatment, the methylation status of MMP-2, $-9,-14,-15$, and -16 was significantly increased, while the remaining MMPs were significantly decreased (Table 2a-2g). Decreased methylation results in greater gene expression [12], so we focused on the correlation between DNA methylation patterns and gene expressions. As shown in Figure 2, MMP-9 demonstrates a hypo-methylated status in KD patients not yet treated with IVIG compared to the control subjects and the KD patients already treated with IVIG. Therefore, the mRNA expression level and DNA methylation of MMP-9 represent a negative correlation, thus indicating that DNA methylation can repress gene 
Table 2a: Methylation patterns of $\mathrm{CpG}$ sites on matrix metallo proteinase between Kawasaki disease patients and control subjects

\begin{tabular}{|c|c|c|c|c|c|c|c|}
\hline Target ID & Symbol & $\begin{array}{l}\text { Fold-Change } \\
\text { (KD1 vs. HC) }\end{array}$ & $\begin{array}{c}p \text {-value } \\
\text { (KD1 vs. HC) }\end{array}$ & $\begin{array}{l}\text { Fold-Change } \\
\text { (KD1 vs. FC) }\end{array}$ & $\begin{array}{c}p \text {-value } \\
\text { (KD1 vs. FC) }\end{array}$ & $\begin{array}{c}\text { Fold-Change } \\
\text { (KD3 vs. KD1) }\end{array}$ & $\begin{array}{c}p \text {-value } \\
\text { (KD3 vs. KD1) }\end{array}$ \\
\hline $\operatorname{cg} 02212280$ & MMP1 & -1.005 & 0.086 & 1.010 & 0.003* & -1.046 & $0.000 *$ \\
\hline $\operatorname{cg} 11531628$ & & -1.001 & 0.652 & -1.003 & 0.206 & 1.005 & $0.029^{*}$ \\
\hline $\operatorname{cg} 13759446$ & & -1.001 & $0.002 *$ & 1.005 & 0.089 & \begin{tabular}{|l|}
-1.017 \\
\end{tabular} & $0.000 *$ \\
\hline cg14543953 & & \begin{tabular}{|l|}
-1.003 \\
\end{tabular} & 0.629 & 1.004 & 0.529 & -1.070 & $0.000^{*}$ \\
\hline $\operatorname{cg} 16575408$ & & -1.002 & 0.488 & 1.002 & 0.408 & -1.014 & $0.000^{*}$ \\
\hline $\operatorname{cg} 18733315$ & & -1.012 & $0.000^{*}$ & -1.001 & 0.708 & -1.003 & 0.389 \\
\hline $\operatorname{cg} 00078334$ & MMP2 & -1.022 & $0.000^{*}$ & -1.018 & $0.000^{*}$ & 1.019 & $0.000^{*}$ \\
\hline cg00087906 & & 1.001 & 0.463 & -1.001 & 0.276 & 1.005 & $0.000^{*}$ \\
\hline $\operatorname{cg} 01821058$ & & -1.003 & 0.071 & -1.004 & $0.041 *$ & 1.007 & $0.000 *$ \\
\hline $\operatorname{cg} 02458945$ & & -1.003 & $0.028^{*}$ & 1.004 & 0.004* & -1.003 & $0.009^{*}$ \\
\hline $\operatorname{cg} 07582157$ & & -1.010 & $0.003 *$ & -1.010 & $0.003 *$ & 1.041 & $0.000 *$ \\
\hline $\operatorname{cg} 08318842$ & & -1.010 & $0.000 *$ & -1.010 & $0.000 *$ & 1.015 & $0.000^{*}$ \\
\hline cg09530163 & & -1.009 & $0.003 *$ & \begin{tabular}{|l|}
-1.009 \\
\end{tabular} & $0.002 *$ & 1.002 & 0.520 \\
\hline $\operatorname{cg} 10079740$ & & -1.003 & $0.015^{*}$ & 1.003 & $0.012 *$ & -1.001 & 0.238 \\
\hline $\operatorname{cg} 12317456$ & & \begin{tabular}{|l|}
-1.011 \\
\end{tabular} & $0.000 *$ & -1.007 & $0.012 *$ & 1.004 & 0.129 \\
\hline cg14400118 & & \begin{tabular}{|l|}
-1.007 \\
\end{tabular} & $0.012 *$ & -1.014 & $0.000 *$ & 1.006 & $0.047^{*}$ \\
\hline cg22950163 & & -1.010 & $0.000 *$ & -1.015 & $0.000 *$ & 1.013 & $0.000 *$ \\
\hline cg25029998 & & -1.011 & 0.263 & -1.003 & 0.793 & -1.008 & 0.408 \\
\hline cg25349472 & & -1.004 & 0.715 & 1.002 & 0.850 & 1.003 & 0.780 \\
\hline $\operatorname{cg} 26795346$ & & -1.007 & $0.024 *$ & 1.002 & 0.568 & -1.018 & $0.000 *$ \\
\hline $\operatorname{cg} 27016988$ & & -1.010 & $0.016^{*}$ & -1.001 & 0.893 & 1.011 & $0.011 *$ \\
\hline cg27279620 & & -1.004 & 0.371 & -1.016 & $0.000 *$ & 1.024 & $0.000 *$ \\
\hline $\operatorname{cg} 27642062$ & & -1.001 & 0.433 & -1.005 & $0.004 *$ & 1.006 & $0.003 *$ \\
\hline $\operatorname{cg} 01027553$ & MMP3 & -1.007 & 0.089 & -1.007 & 0.120 & 1.006 & 0.133 \\
\hline $\operatorname{cg} 03168922$ & & -1.008 & 0.185 & -1.002 & 0.786 & -1.015 & $0.009^{*}$ \\
\hline $\operatorname{cg} 03231596$ & & -1.019 & $0.008^{*}$ & -1.009 & 0.209 & 1.009 & 0.215 \\
\hline $\operatorname{cg} 16466334$ & & -1.009 & $0.017 *$ & -1.010 & $0.007 *$ & -1.014 & $0.000^{*}$ \\
\hline $\operatorname{cg} 17145397$ & & -1.010 & $0.015^{*}$ & 1.002 & 0.613 & -1.012 & $0.007 *$ \\
\hline $\operatorname{cg} 18113270$ & & -1.003 & 0.319 & 1.000 & 0.875 & -1.016 & $0.000^{*}$ \\
\hline $\operatorname{cg} 01813071$ & MMP7 & -1.023 & $0.000 *$ & -1.009 & 0.098 & 1.053 & $0.000^{*}$ \\
\hline $\operatorname{cg} 04059146$ & & -1.010 & $0.020^{*}$ & 1.002 & 0.650 & -1.045 & $0.000^{*}$ \\
\hline $\operatorname{cg} 17707274$ & & -1.047 & $0.000 *$ & -1.043 & $0.000^{*}$ & 1.096 & $0.000^{*}$ \\
\hline cg20645973 & & -1.028 & $0.000 *$ & -1.013 & $0.011 *$ & -1.007 & 0.140 \\
\hline cg24963041 & & -1.002 & 0.516 & 1.007 & 0.063 & -1.025 & $0.000^{*}$ \\
\hline cg25511807 & & -1.137 & $0.000 *$ & -1.064 & $0.001 *$ & 1.053 & $0.005^{*}$ \\
\hline cg01092036 & MMP8 & -1.011 & $0.000^{*}$ & 1.004 & 0.180 & -1.015 & $0.000^{*}$ \\
\hline $\operatorname{cg} 00182727$ & MMP9 & -1.025 & $0.000 *$ & -1.026 & $0.000^{*}$ & 1.017 & $0.000^{*}$ \\
\hline $\operatorname{cg} 00732815$ & & \begin{tabular}{|l|}
-1.047 \\
\end{tabular} & $0.000 *$ & -1.031 & $0.000 *$ & 1.046 & $0.000^{*}$ \\
\hline $\operatorname{cg} 02310296$ & & -1.044 & $0.000 *$ & -1.029 & $0.000 *$ & 1.025 & $0.000^{*}$ \\
\hline $\operatorname{cg} 08176368$ & & 1.004 & 0.127 & 1.010 & $0.001 *$ & -1.024 & $0.000^{*}$ \\
\hline
\end{tabular}


Table 2b: Methylation patterns of CpG sites on matrix metallo proteinase between Kawasaki disease patients and control subjects

\begin{tabular}{|c|c|c|c|c|c|c|c|}
\hline Target ID & Symbol & \begin{tabular}{|l|} 
Fold-Change \\
(KD1 vs. HC)
\end{tabular} & $\begin{array}{c}p \text {-value } \\
\text { (KD1 vs. HC) }\end{array}$ & $\begin{array}{l}\text { Fold-Change } \\
\text { (KD1 vs. FC) } \\
\end{array}$ & $\begin{array}{c}p \text {-value } \\
\text { (KD1 vs. FC) } \\
\end{array}$ & $\begin{array}{c}\text { Fold-Change } \\
\text { (KD3 vs. KD1) }\end{array}$ & $\begin{array}{c}p \text {-value } \\
\text { (KD3 vs. KD1) } \\
\end{array}$ \\
\hline $\operatorname{cg} 10505873$ & MMP9 & -1.091 & $0.000 *$ & -1.057 & $0.000 *$ & 1.064 & $0.000^{*}$ \\
\hline cg20925811 & & -1.049 & $0.000^{*}$ & -1.050 & $0.000 *$ & 1.025 & $0.039 *$ \\
\hline cg21451869 & & -1.024 & $0.000^{*}$ & -1.029 & $0.000 *$ & 1.020 & $0.001 *$ \\
\hline $\operatorname{cg} 23353432$ & & -1.006 & $0.022 *$ & -1.011 & $0.000 *$ & 1.012 & $0.000^{*}$ \\
\hline $\operatorname{cg} 26132320$ & & -1.019 & $0.025^{*}$ & -1.008 & 0.295 & -1.030 & $0.000^{*}$ \\
\hline $\operatorname{cg} 27372994$ & & -1.023 & $0.003^{*}$ & -1.011 & 0.159 & 1.007 & 0.385 \\
\hline $\operatorname{cg} 15259031$ & MMP10 & -1.011 & $0.006^{*}$ & -1.004 & 0.294 & -1.004 & 0.332 \\
\hline cg24960563 & & -1.001 & 0.490 & \begin{tabular}{|l|}
-1.001 \\
\end{tabular} & 0.314 & 1.002 & 0.189 \\
\hline $\operatorname{cg} 00771653$ & MMP11 & -1.019 & $0.002 *$ & 1.002 & 0.744 & -1.046 & $0.000^{*}$ \\
\hline $\operatorname{cg} 03207310$ & & -1.005 & $0.009^{*}$ & 1.000 & 0.798 & 1.004 & $0.032 *$ \\
\hline $\operatorname{cg} 03290040$ & & -1.005 & $0.023 *$ & \begin{tabular}{|l|}
-1.008 \\
\end{tabular} & $0.000 *$ & 1.019 & $0.000^{*}$ \\
\hline $\operatorname{cg} 04058144$ & & -1.001 & 0.335 & -1.003 & 0.054 & 1.004 & $0.002 *$ \\
\hline $\operatorname{cg} 08141518$ & & -1.026 & 0.147 & 1.000 & 0.993 & -1.045 & $0.015^{*}$ \\
\hline $\operatorname{cg} 09133003$ & & -1.005 & \begin{tabular}{|l|l|}
0.476 \\
\end{tabular} & -1.002 & \begin{tabular}{|l|}
0.779 \\
\end{tabular} & \begin{tabular}{|l|}
-1.023 \\
\end{tabular} & $0.004 *$ \\
\hline $\operatorname{cg} 12256538$ & & -1.014 & $0.000^{*}$ & -1.013 & $0.000 *$ & 1.037 & $0.000^{*}$ \\
\hline $\operatorname{cg} 12395479$ & & -1.008 & $0.033^{*}$ & -1.014 & $0.000 *$ & 1.003 & 0.372 \\
\hline cg13361393 & & 1.013 & $0.002 *$ & 1.017 & $0.000 *$ & -1.029 & $0.000^{*}$ \\
\hline $\operatorname{cg} 15808568$ & & -1.007 & 0.759 & 1.041 & 0.065 & -1.018 & 0.412 \\
\hline $\operatorname{cg} 16159491$ & & -1.002 & 0.228 & -1.004 & $0.044 *$ & 1.010 & $0.000^{*}$ \\
\hline $\operatorname{cg} 18240463$ & & 1.003 & 0.695 & -1.002 & 0.771 & -1.015 & $0.025^{*}$ \\
\hline $\operatorname{cg} 19934709$ & & -1.002 & 0.419 & 1.011 & $0.000^{*}$ & -1.012 & $0.000^{*}$ \\
\hline cg20202552 & & -1.009 & $0.000^{*}$ & -1.000 & 0.973 & -1.007 & $0.002 *$ \\
\hline cg21776003 & & 1.007 & $0.009^{*}$ & -1.002 & 0.452 & -1.006 & $0.029^{*}$ \\
\hline cg23261640 & & 1.008 & 0.260 & 1.021 & $0.004 *$ & -1.002 & 0.763 \\
\hline cg23327483 & & 1.004 & $0.028^{*}$ & 1.010 & $0.000 *$ & -1.006 & $0.001 *$ \\
\hline cg27532722 & & -1.030 & $0.000^{*}$ & -1.009 & 0.173 & 1.031 & $0.000^{*}$ \\
\hline $\operatorname{cg} 02368591$ & MMP12 & -1.015 & $0.021^{*}$ & 1.011 & 0.070 & -1.031 & $0.000^{*}$ \\
\hline $\operatorname{cg} 09002726$ & & -1.020 & $0.001 *$ & 1.004 & 0.503 & -1.014 & $0.017 *$ \\
\hline cg14948108 & & -1.008 & $0.038^{*}$ & 1.007 & 0.078 & -1.023 & $0.000^{*}$ \\
\hline cg19304432 & & -1.005 & 0.128 & 1.006 & 0.060 & -1.022 & $0.000^{*}$ \\
\hline cg20487452 & & -1.019 & $0.012 *$ & 1.001 & 0.925 & -1.020 & $0.008^{*}$ \\
\hline cg23979520 & & -1.010 & 0.183 & 1.002 & 0.775 & -1.039 & $0.000 *$ \\
\hline $\operatorname{cg} 03067994$ & MMP13 & -1.009 & $0.005^{*}$ & 1.001 & 0.689 & -1.037 & $0.000^{*}$ \\
\hline $\operatorname{cg} 10085326$ & & -1.007 & 0.439 & 1.005 & 0.526 & -1.067 & $0.000^{*}$ \\
\hline $\operatorname{cg} 13041032$ & & -1.001 & 0.663 & 1.006 & $0.004 *$ & -1.027 & $0.000^{*}$ \\
\hline $\operatorname{cg} 14995062$ & & -1.007 & 0.216 & 1.009 & 0.086 & -1.032 & $0.000^{*}$ \\
\hline $\operatorname{cg} 16919569$ & & -1.018 & $0.028^{*}$ & -1.008 & 0.293 & -1.000 & 0.997 \\
\hline $\operatorname{cg} 19620758$ & & 1.012 & 0.203 & 1.019 & $0.045^{*}$ & -1.049 & $0.000 *$ \\
\hline cg22658979 & & -1.006 & $0.042 *$ & -1.001 & 0.792 & -1.007 & $0.010^{*}$ \\
\hline cg24301681 & & -1.016 & $0.001^{*}$ & -1.009 & $0.031 *$ & -1.018 & $0.000 *$ \\
\hline
\end{tabular}


Table 2c: Methylation patterns of CpG sites on matrix metallo proteinase between Kawasaki disease patients and control subjects

\begin{tabular}{|c|c|c|c|c|c|c|c|}
\hline Target ID & Symbol & $\begin{array}{l}\text { Fold-Change } \\
\text { (KD1 vs. HC) }\end{array}$ & $\begin{array}{c}p \text {-value } \\
\text { (KD1 vs. HC) }\end{array}$ & $\begin{array}{l}\text { Fold-Change } \\
\text { (KD1 vs. FC) }\end{array}$ & $\begin{array}{c}p \text {-value } \\
\text { (KD1 vs. FC) }\end{array}$ & $\begin{array}{c}\text { Fold-Change } \\
\text { (KD3 vs. KD1) }\end{array}$ & $\begin{array}{c}p \text {-value } \\
\text { (KD3 vs. KD1) }\end{array}$ \\
\hline cg00691240 & MMP14 & -1.009 & 0.571 & 1.012 & 0.421 & -1.038 & $0.015^{*}$ \\
\hline $\operatorname{cg} 01016847$ & & -1.051 & $0.000^{*}$ & -1.026 & $0.001 *$ & 1.035 & $0.000 *$ \\
\hline $\operatorname{cg} 01508380$ & & -1.078 & $0.000 *$ & -1.049 & $0.002 *$ & 1.027 & 0.070 \\
\hline $\operatorname{cg} 04556361$ & & -1.001 & 0.885 & 1.012 & $0.009^{*}$ & -1.045 & $0.000 *$ \\
\hline $\operatorname{cg} 05931439$ & & -1.061 & $0.000^{*}$ & -1.033 & $0.005^{*}$ & 1.024 & $0.042 *$ \\
\hline $\operatorname{cg} 08321366$ & & -1.077 & $0.000^{*}$ & -1.059 & $0.000 *$ & 1.023 & $0.039 *$ \\
\hline $\operatorname{cg} 09208010$ & & -1.140 & $0.000^{*}$ & -1.085 & $0.000 *$ & 1.070 & $0.001 *$ \\
\hline $\operatorname{cg} 10418289$ & & -1.081 & $0.000 *$ & -1.057 & $0.000 *$ & 1.090 & $0.000 *$ \\
\hline $\operatorname{cg} 10599444$ & & -1.067 & $0.000^{*}$ & -1.044 & $0.000 *$ & 1.037 & $0.001 *$ \\
\hline $\operatorname{cg} 13094752$ & & -1.085 & $0.000 *$ & -1.049 & $0.001 *$ & 1.007 & 0.594 \\
\hline $\operatorname{cg} 16119835$ & & -1.019 & $0.000 *$ & -1.012 & $0.020 *$ & 1.014 & $0.005^{*}$ \\
\hline $\operatorname{cg} 18525873$ & & -1.004 & 0.436 & 1.005 & 0.331 & 1.002 & 0.638 \\
\hline cg25594542 & & -1.015 & $0.000 *$ & -1.003 & 0.384 & -1.012 & $0.000^{*}$ \\
\hline cg27020028 & & -1.006 & 0.078 & 1.006 & 0.123 & -1.008 & $0.030^{*}$ \\
\hline $\operatorname{cg} 00247629$ & MMP15 & -1.009 & 0.127 & -1.001 & 0.887 & -1.008 & 0.170 \\
\hline $\operatorname{cg} 00963305$ & & 1.002 & 0.095 & -1.004 & $0.000 *$ & 1.004 & $0.000 *$ \\
\hline $\operatorname{cg} 01812337$ & & -1.007 & 0.348 & -1.011 & 0.147 & 1.056 & $0.000 *$ \\
\hline cg04211309 & & -1.016 & $0.000^{*}$ & \begin{tabular}{|l|}
-1.002 \\
\end{tabular} & 0.491 & \begin{tabular}{|l|}
-1.013 \\
\end{tabular} & $0.000 *$ \\
\hline $\operatorname{cg} 05910755$ & & -1.000 & 0.963 & 1.009 & $0.000 *$ & -1.005 & $0.000 *$ \\
\hline $\operatorname{cg} 06649282$ & & -1.022 & $0.000^{*}$ & -1.010 & $0.021 *$ & -1.022 & $0.000^{*}$ \\
\hline $\operatorname{cg} 06856889$ & & -1.008 & $0.003 *$ & -1.000 & 0.878 & 1.006 & $0.026^{*}$ \\
\hline $\operatorname{cg} 08082374$ & & -1.006 & 0.193 & 1.016 & $0.001 *$ & 1.028 & $0.000 *$ \\
\hline $\operatorname{cg} 08514765$ & & -1.011 & $0.000^{*}$ & -1.005 & $0.017 *$ & -1.000 & 0.927 \\
\hline $\operatorname{cg} 08552042$ & & -1.007 & 0.068 & 1.013 & $0.001 *$ & -1.000 & 0.945 \\
\hline $\operatorname{cg} 08877948$ & & -1.005 & 0.215 & -1.009 & $0.037 *$ & 1.026 & $0.000^{*}$ \\
\hline $\operatorname{cg} 08943809$ & & 1.003 & 0.214 & 1.016 & $0.000 *$ & -1.017 & $0.000^{*}$ \\
\hline $\operatorname{cg} 10396713$ & & -1.043 & $0.001 *$ & -1.022 & 0.085 & 1.004 & 0.747 \\
\hline $\operatorname{cg} 16181803$ & & 1.000 & 0.955 & -1.009 & $0.000 *$ & 1.006 & $0.001 *$ \\
\hline $\operatorname{cg} 16652241$ & & 1.001 & 0.616 & -1.005 & $0.018 *$ & 1.008 & $0.000^{*}$ \\
\hline $\operatorname{cg} 18333626$ & & -1.001 & 0.793 & -1.004 & 0.451 & 1.011 & $0.040^{*}$ \\
\hline $\operatorname{cg} 18442019$ & & -1.009 & $0.041^{*}$ & -1.006 & 0.141 & 1.007 & 0.100 \\
\hline cg20566643 & & -1.006 & $0.001 *$ & -1.010 & $0.000 *$ & 1.013 & $0.000^{*}$ \\
\hline cg20751926 & & -1.020 & $0.013^{*}$ & -1.022 & $0.008^{*}$ & -1.005 & 0.524 \\
\hline cg24306779 & & -1.001 & 0.811 & -1.011 & $0.000 *$ & 1.004 & 0.154 \\
\hline $\operatorname{cg} 25449950$ & & 1.015 & 0.095 & -1.002 & 0.856 & 1.004 & 0.657 \\
\hline cg26725183 & & -1.003 & 0.335 & 1.017 & $0.000 *$ & 1.011 & $0.001 *$ \\
\hline cg26919014 & & -1.018 & $0.000 *$ & -1.004 & 0.225 & -1.004 & 0.233 \\
\hline cg27208052 & & -1.033 & $0.000 *$ & -1.006 & 0.448 & \begin{tabular}{|l|}
1.019 \\
\end{tabular} & $0.017 *$ \\
\hline $\operatorname{cg} 00021786$ & MMP16 & -1.009 & 0.169 & -1.008 & 0.210 & 1.011 & 0.105 \\
\hline $\operatorname{cg} 02889488$ & & 1.001 & 0.796 & 1.026 & $0.000 *$ & -1.018 & $0.000^{*}$ \\
\hline
\end{tabular}


Table 2d: Methylation patterns of CpG sites on matrix metallo proteinase between Kawasaki disease patients and control subjects

\begin{tabular}{|c|c|c|c|c|c|c|c|}
\hline Target ID & Symbol & $\begin{array}{l}\text { Fold-Change } \\
\text { (KD1 vs. HC) }\end{array}$ & $\begin{array}{c}p \text {-value } \\
\text { (KD1 vs. HC) }\end{array}$ & $\begin{array}{l}\text { Fold-Change } \\
\text { (KD1 vs. FC) }\end{array}$ & $\begin{array}{c}p \text {-value } \\
(\text { KD1 vs. FC) }\end{array}$ & $\begin{array}{c}\text { Fold-Change } \\
\text { (KD3 vs. KD1) }\end{array}$ & $\begin{array}{c}p \text {-value } \\
\text { (KD3 vs. KD1) }\end{array}$ \\
\hline $\operatorname{cg} 03210866$ & MMP16 & -1.019 & 0.277 & 1.013 & 0.437 & -1.030 & 0.090 \\
\hline $\operatorname{cg} 04684553$ & & 1.016 & $0.018^{*}$ & 1.008 & 0.205 & 1.070 & $0.000^{*}$ \\
\hline $\operatorname{cg} 05033271$ & & 1.006 & 0.383 & 1.000 & 0.981 & 1.107 & $0.000^{*}$ \\
\hline cg07609388 & & -1.006 & $0.029^{*}$ & -1.026 & $0.000^{*}$ & 1.019 & $0.000^{*}$ \\
\hline $\operatorname{cg} 08624180$ & & \begin{tabular}{|l|}
-1.007 \\
\end{tabular} & $0.050^{*}$ & -1.011 & $0.003 *$ & 1.018 & $0.000^{*}$ \\
\hline $\operatorname{cg} 11536457$ & & \begin{tabular}{|l|}
-1.001 \\
\end{tabular} & 0.685 & 1.000 & 0.935 & 1.025 & $0.000^{*}$ \\
\hline $\operatorname{cg} 14644752$ & & 1.001 & 0.781 & 1.015 & $0.000 *$ & 1.033 & $0.000^{*}$ \\
\hline $\operatorname{cg} 16852892$ & & -1.007 & $0.005^{*}$ & -1.006 & $0.032 *$ & 1.010 & $0.000^{*}$ \\
\hline $\operatorname{cg} 22405982$ & & -1.004 & 0.078 & 1.002 & \begin{tabular}{|l|}
0.460 \\
\end{tabular} & \begin{tabular}{|l|}
-1.001 \\
\end{tabular} & 0.470 \\
\hline $\operatorname{cg} 24403959$ & & -1.003 & 0.544 & -1.010 & 0.082 & -1.002 & 0.763 \\
\hline $\operatorname{cg} 24456365$ & & -1.006 & 0.112 & -1.004 & 0.266 & -1.011 & $0.008^{*}$ \\
\hline $\operatorname{cg} 26075830$ & & -1.021 & $0.000^{*}$ & -1.008 & 0.059 & 1.003 & 0.442 \\
\hline $\operatorname{cg} 02309230$ & MMP17 & 1.016 & 0.109 & 1.032 & $0.002 *$ & 1.003 & 0.756 \\
\hline $\operatorname{cg} 02714882$ & & -1.002 & 0.097 & 1.002 & 0.055 & -1.002 & 0.065 \\
\hline $\operatorname{cg} 03064145$ & & -1.004 & $0.048^{*}$ & -1.011 & $0.000 *$ & 1.007 & $0.003^{*}$ \\
\hline $\operatorname{cg} 04346459$ & & -1.036 & $0.045^{*}$ & -1.002 & 0.907 & 1.028 & 0.114 \\
\hline $\operatorname{cg} 07699454$ & & -1.022 & $0.016^{*}$ & 1.001 & 0.863 & 1.016 & 0.067 \\
\hline $\operatorname{cg} 10105149$ & & -1.008 & $0.037^{*}$ & -1.007 & 0.065 & -1.004 & 0.308 \\
\hline $\operatorname{cg} 10673839$ & & 1.003 & 0.487 & 1.003 & 0.468 & -1.015 & $0.000^{*}$ \\
\hline $\operatorname{cg} 11510557$ & & 1.000 & 0.720 & 1.009 & $0.000^{*}$ & -1.010 & $0.000^{*}$ \\
\hline $\operatorname{cg} 13082201$ & & -1.002 & 0.229 & -1.006 & $0.002 *$ & 1.009 & $0.000^{*}$ \\
\hline $\operatorname{cg} 14215776$ & & 1.001 & 0.131 & -1.003 & $0.000^{*}$ & 1.006 & $0.000^{*}$ \\
\hline $\operatorname{cg} 15454599$ & & -1.006 & $0.004 *$ & -1.006 & $0.002 *$ & 1.006 & $0.005^{*}$ \\
\hline $\operatorname{cg} 20393882$ & & -1.007 & 0.248 & 1.009 & 0.147 & -1.000 & 0.981 \\
\hline cg20844545 & & -1.000 & 0.838 & -1.005 & $0.001 *$ & 1.004 & $0.009^{*}$ \\
\hline $\operatorname{cg} 23477406$ & & -1.001 & 0.563 & -1.004 & $0.000^{*}$ & 1.004 & $0.000^{*}$ \\
\hline $\operatorname{cg} 24493940$ & & -1.003 & 0.550 & 1.000 & 0.930 & -1.021 & $0.000^{*}$ \\
\hline $\operatorname{cg} 25531700$ & & -1.004 & 0.060 & -1.009 & $0.000^{*}$ & 1.014 & $0.000^{*}$ \\
\hline $\operatorname{cg} 26326372$ & & -1.021 & 0.153 & -1.032 & $0.031 *$ & 1.020 & 0.172 \\
\hline $\operatorname{cg} 26356412$ & & -1.019 & $0.002 *$ & -1.016 & $0.009 *$ & 1.023 & $0.000^{*}$ \\
\hline cg26569074 & & -1.009 & $0.001 *$ & -1.017 & $0.000 *$ & -1.004 & 0.133 \\
\hline $\operatorname{cg} 27207041$ & & 1.001 & 0.657 & 1.006 & $0.005^{*}$ & -1.002 & 0.391 \\
\hline $\operatorname{cg} 27305698$ & & -1.006 & $0.045^{*}$ & 1.002 & 0.559 & -1.008 & $0.006^{*}$ \\
\hline $\operatorname{cg} 03509949$ & MMP19 & -1.160 & $0.000^{*}$ & -1.096 & $0.000^{*}$ & 1.126 & $0.000^{*}$ \\
\hline $\operatorname{cg} 09907201$ & & -1.023 & $0.001 *$ & -1.018 & $0.008^{*}$ & -1.029 & $0.000^{*}$ \\
\hline $\operatorname{cg} 16936370$ & & -1.021 & $0.015^{*}$ & -1.019 & $0.022 *$ & 1.030 & $0.001 *$ \\
\hline $\operatorname{cg} 17865265$ & & -1.124 & $0.000^{*}$ & -1.068 & $0.000^{*}$ & 1.074 & $0.000^{*}$ \\
\hline $\operatorname{cg} 18787783$ & & -1.005 & 0.201 & 1.004 & 0.322 & -1.036 & $0.000^{*}$ \\
\hline $\operatorname{cg} 25006073$ & & -1.010 & 0.116 & 1.003 & 0.615 & -1.095 & $0.000^{*}$ \\
\hline $\operatorname{cg} 10553219$ & MMP20 & -1.007 & 0.684 & 1.043 & $0.028^{*}$ & -1.020 & 0.284 \\
\hline
\end{tabular}


Table 2e: Methylation patterns of $\mathrm{CpG}$ sites on matrix metallo proteinase between Kawasaki disease patients and control subjects

\begin{tabular}{|c|c|c|c|c|c|c|c|}
\hline Target ID & Symbol & $\begin{array}{c}\text { Fold-Change } \\
\text { (KD1 vs. HC) }\end{array}$ & $\begin{array}{c}p \text {-value } \\
\text { (KD1 vs. HC) }\end{array}$ & $\begin{array}{l}\text { Fold-Change } \\
\text { (KD1 vs. FC) }\end{array}$ & $\begin{array}{c}p \text {-value } \\
\text { (KD1 vs. FC) }\end{array}$ & $\begin{array}{c}\text { Fold-Change } \\
\text { (KD3 vs. KD1) }\end{array}$ & $\begin{array}{c}p \text {-value } \\
\text { (KD3 vs. KD1) }\end{array}$ \\
\hline $\operatorname{cg} 12020179$ & MMP20 & -1.003 & 0.505 & 1.021 & $0.000^{*}$ & -1.047 & $0.000 *$ \\
\hline $\operatorname{cg} 23275776$ & & 1.001 & 0.889 & -1.005 & 0.247 & -1.076 & $0.000^{*}$ \\
\hline cg26757793 & & -1.004 & 0.386 & 1.011 & $0.018^{*}$ & -1.025 & $0.000 *$ \\
\hline $\operatorname{cg} 02964396$ & MMP21 & 1.002 & 0.142 & 1.001 & 0.628 & 1.001 & 0.586 \\
\hline $\operatorname{cg} 03437479$ & & -1.020 & $0.007 *$ & -1.006 & 0.359 & 1.011 & 0.120 \\
\hline $\operatorname{cg} 03553576$ & & -1.019 & 0.066 & -1.011 & 0.276 & -1.006 & 0.567 \\
\hline $\operatorname{cg} 04358037$ & & -1.009 & $0.006 *$ & -1.004 & 0.240 & -1.010 & $0.002 *$ \\
\hline $\operatorname{cg} 05670995$ & & -1.041 & $0.015^{*}$ & -1.040 & $0.018^{*}$ & -1.015 & 0.346 \\
\hline $\operatorname{cg} 05721877$ & & 1.053 & 0.236 & 1.039 & 0.386 & -1.096 & $0.039 *$ \\
\hline $\operatorname{cg} 07954108$ & & -1.008 & $0.018^{*}$ & -1.012 & $0.001^{*}$ & -1.006 & 0.084 \\
\hline $\operatorname{cg} 09540803$ & & -1.001 & 0.603 & -1.003 & 0.257 & -1.019 & $0.000^{*}$ \\
\hline $\operatorname{cg} 11240212$ & & -1.001 & 0.336 & -1.003 & $0.035^{*}$ & 1.003 & 0.088 \\
\hline $\operatorname{cg} 11254426$ & & -1.011 & $0.044^{*}$ & 1.001 & 0.861 & -1.017 & $0.003 *$ \\
\hline $\operatorname{cg} 12666819$ & & -1.004 & 0.100 & -1.003 & 0.313 & 1.002 & 0.395 \\
\hline $\operatorname{cg} 12854248$ & & -1.015 & $0.000^{*}$ & -1.008 & $0.010^{*}$ & 1.000 & 0.960 \\
\hline $\operatorname{cg} 24307114$ & & -1.011 & $0.001 *$ & -1.013 & $0.000^{*}$ & 1.019 & $0.000^{*}$ \\
\hline cg24383498 & & -1.001 & 0.825 & 1.004 & 0.552 & -1.023 & $0.000 *$ \\
\hline $\operatorname{cg} 25400200$ & & -1.013 & $0.044^{*}$ & -1.012 & 0.060 & -1.026 & $0.000^{*}$ \\
\hline cg25906360 & & -1.013 & 0.059 & -1.006 & 0.349 & -1.032 & $0.000 *$ \\
\hline $\operatorname{cg} 26907302$ & & -1.009 & $0.005^{*}$ & -1.003 & 0.388 & -1.007 & $0.030^{*}$ \\
\hline $\operatorname{cg} 00262621$ & MMP23A & 1.021 & $0.002 *$ & 1.010 & 0.130 & -1.031 & $0.000 *$ \\
\hline $\operatorname{cg} 00297832$ & & 1.029 & $0.005^{*}$ & 1.028 & $0.007^{*}$ & -1.003 & 0.729 \\
\hline $\operatorname{cg} 00341415$ & & -1.014 & $0.012^{*}$ & -1.004 & 0.435 & -1.016 & $0.005^{*}$ \\
\hline $\operatorname{cg} 00369151$ & & 1.004 & 0.807 & -1.005 & 0.769 & -1.025 & 0.179 \\
\hline $\operatorname{cg} 01154903$ & & -1.009 & 0.224 & -1.007 & 0.332 & -1.017 & $0.028^{*}$ \\
\hline $\operatorname{cg} 02519897$ & & 1.002 & 0.705 & 1.001 & 0.818 & 1.018 & $0.003 *$ \\
\hline $\operatorname{cg} 02599583$ & & -1.006 & 0.762 & 1.022 & 0.267 & -1.044 & $0.035^{*}$ \\
\hline $\operatorname{cg} 03001942$ & & -1.011 & $0.020^{*}$ & -1.006 & 0.182 & 1.008 & 0.092 \\
\hline $\operatorname{cg} 03136646$ & & -1.029 & $0.000^{*}$ & -1.018 & $0.001^{*}$ & 1.021 & $0.000 *$ \\
\hline $\operatorname{cg} 03427058$ & & 1.011 & 0.512 & 1.051 & $0.003 *$ & -1.083 & $0.000 *$ \\
\hline $\operatorname{cg} 03930970$ & & -1.005 & 0.076 & 1.000 & 0.892 & -1.002 & 0.387 \\
\hline $\operatorname{cg} 04437922$ & & -1.011 & 0.102 & 1.001 & 0.864 & -1.054 & $0.000 *$ \\
\hline $\operatorname{cg} 04882394$ & & -1.000 & 0.986 & -1.012 & $0.000^{*}$ & 1.014 & $0.000 *$ \\
\hline $\operatorname{cg} 05642789$ & & 1.011 & 0.561 & 1.060 & $0.003 *$ & -1.072 & $0.000 *$ \\
\hline $\operatorname{cg} 06084034$ & & -1.107 & $0.012^{*}$ & -1.040 & 0.318 & 1.019 & 0.639 \\
\hline $\operatorname{cg} 06750152$ & & -1.009 & $0.024 *$ & 1.007 & 0.073 & -1.012 & $0.006^{*}$ \\
\hline $\operatorname{cg} 09937190$ & & -1.105 & $0.001 *$ & -1.066 & $0.036^{*}$ & 1.038 & 0.217 \\
\hline $\operatorname{cg} 11648594$ & & 1.019 & $0.007^{*}$ & 1.015 & $0.032 *$ & -1.028 & $0.000 *$ \\
\hline $\operatorname{cg} 12534645$ & & 1.025 & $0.042 *$ & 1.017 & 0.161 & 1.016 & 0.191 \\
\hline $\operatorname{cg} 17402325$ & & -1.016 & $0.009^{*}$ & -1.017 & $0.005^{*}$ & 1.008 & 0.186 \\
\hline
\end{tabular}


Table 2f: Methylation patterns of CpG sites on matrix metallo proteinase between Kawasaki disease patients and control subjects

\begin{tabular}{|c|c|c|c|c|c|c|c|}
\hline Target ID & Symbol & $\begin{array}{c}\text { Fold-Change } \\
\text { (KD1 vs. HC) } \\
\end{array}$ & $\begin{array}{c}p \text {-value } \\
\text { (KD1 vs. HC) }\end{array}$ & $\begin{array}{l}\text { Fold-Change } \\
\text { (KD1 vs. FC) }\end{array}$ & $\begin{array}{c}p \text {-value } \\
\text { (KD1 vs. FC) } \\
\end{array}$ & $\begin{array}{c}\text { Fold-Change } \\
\text { (KD3 vs. KD1) }\end{array}$ & $\begin{array}{c}p \text {-value } \\
\text { (KD3 vs. KD1) } \\
\end{array}$ \\
\hline cg18852096 & MMP23A & -1.010 & 0.475 & 1.003 & 0.840 & -1.051 & $0.000^{*}$ \\
\hline cg21265770 & & -1.018 & $0.027 *$ & 1.002 & 0.812 & -1.034 & $0.000^{*}$ \\
\hline cg23125449 & & -1.009 & $0.013 *$ & -1.003 & 0.464 & 1.011 & $0.006^{*}$ \\
\hline cg24688574 & & -1.001 & 0.724 & 1.005 & 0.140 & -1.032 & $0.000^{*}$ \\
\hline cg27474382 & & 1.013 & 0.219 & 1.017 & 0.113 & -1.011 & 0.277 \\
\hline cg00262621 & MMP23B & 1.021 & $0.002 *$ & 1.010 & 0.130 & -1.031 & $0.000 *$ \\
\hline cg00297832 & & 1.029 & $0.005^{*}$ & 1.028 & $0.007 *$ & -1.003 & 0.729 \\
\hline cg00369151 & & 1.004 & 0.807 & -1.005 & 0.769 & -1.025 & 0.179 \\
\hline cg01154903 & & -1.009 & 0.224 & -1.007 & 0.332 & -1.017 & $0.028^{*}$ \\
\hline cg02599583 & & -1.006 & 0.762 & 1.022 & 0.267 & -1.044 & $0.035^{*}$ \\
\hline cg03001942 & & -1.011 & $0.020 *$ & -1.006 & 0.182 & 1.008 & 0.092 \\
\hline cg03136646 & & -1.029 & $0.000 *$ & -1.018 & $0.001 *$ & 1.021 & $0.000 *$ \\
\hline $\operatorname{cg} 03427058$ & & 1.011 & 0.512 & 1.051 & $0.003^{*}$ & -1.083 & $0.000 *$ \\
\hline cg03930970 & & -1.005 & 0.076 & 1.000 & 0.892 & -1.002 & 0.387 \\
\hline cg04854189 & & -1.008 & $0.030 *$ & -1.003 & 0.348 & -1.020 & $0.000 *$ \\
\hline cg04882394 & & -1.000 & 0.986 & -1.012 & $0.000 *$ & 1.014 & $0.000 *$ \\
\hline cg05642789 & & 1.011 & 0.561 & 1.060 & $0.003 *$ & -1.072 & $0.000 *$ \\
\hline cg06080300 & & -1.068 & $0.000 *$ & -1.043 & $0.000 *$ & 1.026 & $0.010^{*}$ \\
\hline cg06084034 & & -1.107 & $0.012 *$ & -1.040 & 0.318 & 1.019 & 0.639 \\
\hline cg06912282 & & -1.115 & $0.000 *$ & -1.059 & $0.000^{*}$ & 1.076 & $0.000^{*}$ \\
\hline cg09937190 & & -1.105 & $0.001 *$ & -1.066 & $0.036^{*}$ & 1.038 & 0.217 \\
\hline cg11648594 & & 1.019 & $0.007 *$ & 1.015 & $0.032 *$ & -1.028 & $0.000 *$ \\
\hline $\operatorname{cg} 12534645$ & & 1.025 & $0.042 *$ & 1.017 & 0.161 & 1.016 & 0.191 \\
\hline $\operatorname{cg} 17402325$ & & -1.016 & $0.009 *$ & -1.017 & $0.005 *$ & 1.008 & 0.186 \\
\hline cg18852096 & & -1.010 & 0.475 & 1.003 & 0.840 & -1.051 & $0.000 *$ \\
\hline $\operatorname{cg} 23125449$ & & -1.009 & $0.013 *$ & -1.003 & 0.464 & 1.011 & $0.006^{*}$ \\
\hline cg02288791 & MMP24 & -1.005 & 0.280 & 1.003 & 0.421 & -1.035 & $0.000 *$ \\
\hline cg03260792 & & 1.000 & 0.806 & -1.002 & 0.252 & 1.001 & 0.522 \\
\hline $\operatorname{cg} 04316754$ & & -1.013 & 0.056 & 1.001 & 0.832 & 1.046 & $0.000 *$ \\
\hline $\operatorname{cg} 12483876$ & & -1.003 & 0.676 & -1.007 & 0.289 & 1.056 & $0.000 *$ \\
\hline cg15270813 & & -1.017 & 0.088 & -1.005 & 0.610 & 1.039 & $0.000^{*}$ \\
\hline cg00456894 & MMP25 & 1.000 & 0.838 & 1.004 & $0.000^{*}$ & 1.000 & 0.716 \\
\hline cg01270736 & & -1.021 & $0.002 *$ & -1.026 & $0.000^{*}$ & 1.005 & 0.448 \\
\hline cg02616220 & & -1.005 & 0.231 & 1.011 & $0.008^{*}$ & -1.048 & $0.000^{*}$ \\
\hline cg02988422 & & -1.014 & 0.123 & -1.013 & 0.152 & 1.009 & 0.325 \\
\hline cg05168200 & & 1.004 & 0.330 & 1.020 & $0.000^{*}$ & -1.030 & $0.000 *$ \\
\hline cg06697694 & & 1.003 & 0.172 & 1.002 & 0.264 & 1.020 & $0.000 *$ \\
\hline cg08607821 & & -1.008 & 0.095 & -1.005 & 0.280 & -1.005 & 0.285 \\
\hline $\operatorname{cg} 10620273$ & & 1.001 & 0.654 & 1.007 & $0.001 *$ & -1.004 & $0.040 *$ \\
\hline $\operatorname{cg} 16856286$ & & -1.002 & 0.437 & -1.004 & 0.211 & 1.008 & $0.016^{*}$ \\
\hline
\end{tabular}


Table 2g: Methylation patterns of CpG sites on matrix metallo proteinase between Kawasaki disease patients and control subjects

\begin{tabular}{|c|c|c|c|c|c|c|c|}
\hline Target ID & Symbol & $\begin{array}{l}\text { Fold-Change } \\
\text { (KD1 vs. HC) }\end{array}$ & $\begin{array}{c}p \text {-value } \\
\text { (KD1 vs. HC) }\end{array}$ & $\begin{array}{l}\text { Fold-Change } \\
\text { (KD1 vs. FC) }\end{array}$ & $\begin{array}{c}p \text {-value } \\
\text { (KD1 vs. FC) }\end{array}$ & $\begin{array}{c}\text { Fold-Change } \\
\text { (KD3 vs. KD1) }\end{array}$ & $\begin{array}{c}p \text {-value } \\
\text { (KD3 vs. KD1) }\end{array}$ \\
\hline $\operatorname{cg} 19612376$ & MMP25 & -1.004 & 0.237 & 1.001 & 0.789 & -1.003 & 0.295 \\
\hline $\operatorname{cg} 22053861$ & & -1.004 & 0.061 & -1.002 & 0.255 & -1.001 & 0.490 \\
\hline cg23531828 & & 1.002 & 0.867 & -1.012 & 0.181 & -1.014 & 0.125 \\
\hline $\operatorname{cg} 25584355$ & & 1.001 & 0.585 & -1.005 & $0.008^{*}$ & 1.001 & 0.496 \\
\hline $\operatorname{cg} 26705553$ & & -1.006 & $0.001 *$ & 1.009 & $0.000 *$ & -1.003 & 0.172 \\
\hline cg26942943 & & -1.006 & $0.006^{*}$ & -1.002 & 0.444 & 1.010 & $0.000^{*}$ \\
\hline $\operatorname{cg} 02706104$ & MMP26 & -1.005 & 0.147 & 1.000 & 0.990 & -1.038 & $0.000^{*}$ \\
\hline $\operatorname{cg} 05316601$ & & -1.013 & $0.000 *$ & -1.003 & 0.334 & -1.030 & $0.000^{*}$ \\
\hline $\operatorname{cg} 09805271$ & & -1.013 & 0.176 & -1.009 & 0.335 & -1.104 & $0.000^{*}$ \\
\hline $\operatorname{cg} 12493906$ & & -1.028 & $0.000 *$ & -1.008 & 0.143 & 1.017 & $0.002 *$ \\
\hline $\operatorname{cg} 17992177$ & & -1.018 & $0.001 *$ & -1.006 & 0.261 & -1.032 & $0.000^{*}$ \\
\hline cg23389785 & & -1.009 & $0.015^{*}$ & -1.006 & 0.108 & 1.000 & 0.943 \\
\hline $\operatorname{cg} 06259570$ & MMP27 & -1.012 & $0.012 *$ & -1.011 & $0.019^{*}$ & -1.037 & $0.000^{*}$ \\
\hline cg09758894 & & -1.011 & $0.015^{*}$ & 1.008 & 0.082 & -1.015 & $0.001^{*}$ \\
\hline $\operatorname{cg} 13252516$ & & 1.003 & 0.329 & 1.006 & 0.080 & -1.038 & $0.000^{*}$ \\
\hline $\operatorname{cg} 18932968$ & & -1.003 & 0.302 & 1.013 & $0.000 *$ & -1.025 & $0.000^{*}$ \\
\hline cg25277638 & & -1.024 & $0.039 *$ & -1.009 & 0.456 & 1.004 & 0.730 \\
\hline $\operatorname{cg} 01134296$ & MMP28 & -1.024 & $0.000 *$ & -1.019 & $0.000 *$ & 1.020 & $0.000^{*}$ \\
\hline $\operatorname{cg} 01352921$ & & -1.001 & 0.304 & -1.002 & 0.201 & 1.004 & $0.001^{*}$ \\
\hline $\operatorname{cg} 01622018$ & & -1.062 & $0.000 *$ & -1.022 & $0.046^{*}$ & 1.018 & 0.091 \\
\hline cg04790749 & & -1.004 & 0.614 & 1.009 & 0.273 & -1.063 & $0.000^{*}$ \\
\hline $\operatorname{cg} 07330075$ & & -1.005 & 0.184 & 1.009 & $0.021^{*}$ & -1.029 & $0.000^{*}$ \\
\hline $\operatorname{cg} 07546558$ & & 1.009 & $0.002 *$ & 1.008 & $0.003 *$ & -1.011 & $0.000^{*}$ \\
\hline $\operatorname{cg} 07916589$ & & -1.005 & 0.231 & 1.006 & 0.164 & 1.004 & 0.378 \\
\hline $\operatorname{cg} 09357580$ & & 1.004 & $0.004 *$ & 1.003 & $0.031 *$ & -1.003 & $0.018^{*}$ \\
\hline $\operatorname{cg} 09730211$ & & 1.003 & 0.143 & 1.007 & $0.003 *$ & -1.014 & $0.000^{*}$ \\
\hline cg09807148 & & -1.014 & $0.000^{*}$ & -1.002 & 0.444 & -1.002 & 0.501 \\
\hline $\operatorname{cg} 12700449$ & & -1.028 & $0.000^{*}$ & -1.017 & $0.001 *$ & -1.001 & 0.893 \\
\hline $\operatorname{cg} 13326172$ & & 1.001 & 0.460 & -1.005 & $0.001 *$ & 1.005 & $0.001 *$ \\
\hline $\operatorname{cg} 16246294$ & & 1.002 & 0.486 & 1.014 & $0.000 *$ & -1.015 & $0.000^{*}$ \\
\hline $\operatorname{cg} 25172682$ & & 1.018 & 0.095 & 1.009 & 0.415 & -1.042 & $0.000^{*}$ \\
\hline
\end{tabular}

For simplicity, we listed the $\mathrm{p}$ values only to $10-3$. Actually, the $\mathrm{CpG}$ marker cg10505873 (the target for further examination) has $\mathrm{p}$ value $<10-5$ among all of the three comparisons (KD1 vs. FC, KD1 vs. HC and KD3 vs. KD1). When examined with the FDR (with Bonferroni correction), cg10505873 is also significant among the three comparisons.

KD1: Kawasaki disease before IVIG treatment; KD3: Kawasaki disease > 3 weeks after IVIG treatment; FC: febrile control; HC: healthy control. 
expression.

\section{MMP-8, -9 and -25 expressions in peripheral white blood cells (WBCs) of KD patients and controls}

We examined the mRNA levels of MMP-8, -9 , and -25 in a separate cohort consisting of $31 \mathrm{KD}$ patients and 46 controls ( 23 healthy and 23 febrile controls) using realtime PCR. We found higher MMP-8 ( $p=0.004)$, MMP$9(p=0.024)$, and MMP-25 mRNA levels $(p=0.002)$ in the WBCs of KD patients than in those of the controls, as shown in Figure 3. Such findings are consistent with the Affymetrix GeneChip ${ }^{\circledR}$ Human Transcriptome Array 2.0 results. Moreover, the mRNA level of MMP-9 was greater in $\mathrm{KD}$ patients who developed CAL than those who did not $(p=0.035)$ (Figure 4).

\section{DISCUSSION}

To the best of our knowledge, ours is the first study to comprehensively survey global DNA methylation levels and transcripts of MMPs between KD patients and control subjects. Our noteworthy observations include the epigenetic hypomethylation and upregulation of MMP-9 in KD, as well as the increase of mRNA levels of MMP-9 in $\mathrm{KD}$ patients with CAL formation.

Cardiovascular complications are the leading cause of morbidity and mortality in KD patients [14]. Coronary dilatation followed by aneurysm occurs in $20-25 \%$ of KD children who do not receive treatment $[15,16]$. Undergoing IVIG treatment can effectively decrease the likelihood of CAA formation, but its effect

a

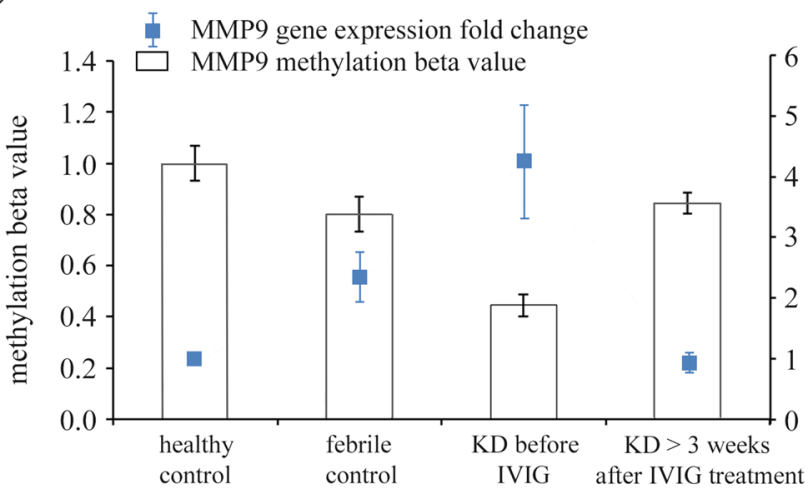

on etiology aneurysm formation remains unclear $[14,17]$. An increasing amount of evidence has also suggested that children with KD are at a long-term risk for subsequent cardiovascular events, possibly due to the endothelial dysfunction and inflammation of the coronary and systemic arteries [18, 19]. MMPs have an important controlling influence regarding maintaining coronary vascular wall structure and function [4]. Previous studies have reported a significant correlation between MMP9 with coronary artery disease [20] and outcomes after acute myocardial infarction [21]. The overexpression of MMP-2, -8, -9, and -12 was related to abdominal aortic aneurysm [22]. Likewise, MMP-8, -9 , and -12 seem to be associated with collagen I and collagen III, as well as their degradation products and vascular remodeling [22]. More importantly, Newby et al. proposed that MMP-mediated macrophage invasion and tissue destruction fosters the ideal environment for atherosclerotic plaque rupture [23].

While increasing evidence has indicated that cytokine profiles are related to the pathogenesis of $\mathrm{KD}$, the actual CALs involved remain unclear. MMP-3 and -12 haplotypes have been correlated with CAA formation in patients with KD [7], while MMP-11 polymorphism may be correlated with KD in Korean children [24]. Furthermore, diffuse and strong staining of MMP-9 was observed in coronary lesions of children who died from KD [25], as was an increased MMP-9 expression in vascular endothelial cells induced by the serum of KD patients [26]. Remarkably, MMP-9 activity can aid in breaking down elastin [27], so hampering such activity can improve coronary artery inflammation in a KD animal model [28]. IVIG treatment can also inhibit monocytes that express MMP-9, thus decreasing chemotactic migration of monocytes and CAL development [29]. was

b

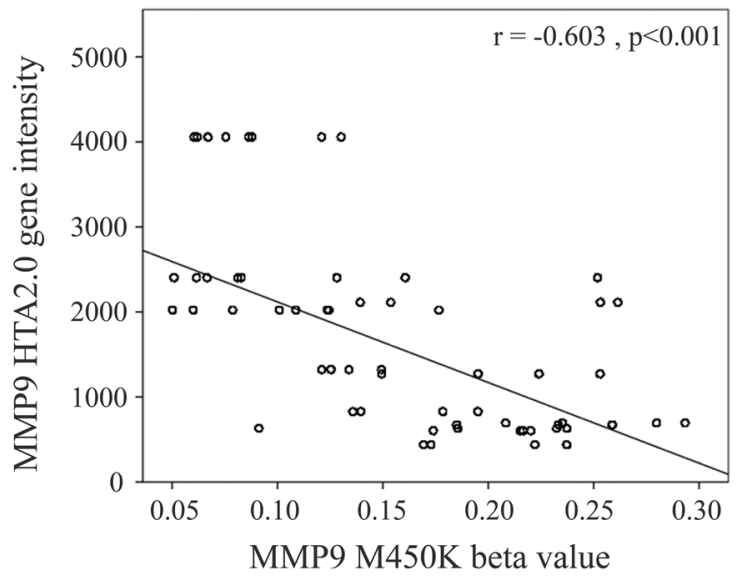

Figure 2: Integration of CpG marker (cg10505873) methylation pattern and gene expression profile of matrix metalloproteinase (MMP)-9. a. The methylation patterns of the representative CpG marker and gene expression profile of MMP-9 showed negative tendencies and were observed to change in both the healthy and febrile control subjects, as well as KD patients before and after receiving intravenous immunoglobulin treatment. The histogram and curve are presented as mean \pm standard error. $\mathbf{b}$. We used scatter plots to demonstrate the correlations between mRNA levels and DNA methylation, which show that mRNA levels were negatively correlated with DNA methylation (Pearson's correlation coefficient approximately -0.603 and $p<0.001$ ). 
Table 3: Basal characteristics of controls and patients with KD

\begin{tabular}{|l|c|l|c|}
\hline Characteristic & $\begin{array}{l}\text { Healthy controls } \\
(\boldsymbol{n}=\mathbf{4 1})\end{array}$ & $\begin{array}{l}\text { Febrile controls } \\
(\boldsymbol{n}=\mathbf{4 1})\end{array}$ & $\begin{array}{l}\text { Patients with KD } \\
(\boldsymbol{n}=\mathbf{4 9})\end{array}$ \\
\hline Male gender, n (\%) & $26(63.4)$ & $25(60.9)$ & $34(69.3)$ \\
\hline Mean $( \pm$ SD), age $(\mathrm{y})$ & $6.1 \pm 4.7$ & $3.3 \pm 2.6$ & $1.8 \pm 1.7$ \\
\hline CAL formation & & & $18(36.7 \%)$ \\
\hline
\end{tabular}

CAL, coronary artery lesion; KD, Kawasaki disease; SD, standard deviation

Another study showed that treatment with TNF- $\alpha$ [30] and advanced glycation end products decrease the promoter DNA methylation and increase the endogenous expression of MMP-9 in the human keratinocyte cell line [31]. Moreover, oxidized low-density lipoprotein significantly increased primary human aortic smooth muscle cell migration by decreasing DNA methylation levels and increasing MMP-9 expression [32], which supports our finding that expression abundance of MMP-9 can be altered and negatively correlated with the methylation profile. In our previous studies, we have described KD patients showing remarkably increased mRNA expression in TLRs and hypomethylation at TLR gene promoters [13]. Interestingly, Soria-Valles at al. showed that MMP25 could activate the innate immune response [33]. Similarly, our study has also found increased transcripts of MMP-8, MMP-9, and MMP-25 in KD, indicating that these proteinases may be potential therapeutic targets for KD.

Prostaglandin E2 (PGE2) is a prostanoid created by almost all vascular cell types and takes part in vascular wall remodeling by regulating MMP activities [34]. PGE2 can further stimulate MMP-9 expression in inflammatory cells [35], and we discovered that plasma PGE2 is correlated with the prevention of IVIG resistance and CAL formation through CD40L in KD [36]. A previous study found that transcripts correlated with MMP-9 and interferon-gamma in PBMC in patients with coronary artery ectasia [37], while we observed an association among IFNG gene polymorphisms, susceptibility to KD, IVIG responsiveness, and plasma IFN- $\gamma$ levels in KD patients [38]. In this study, we demonstrated that MMP-9 was not only upregulated in KD but also had a significant correlation with CAL formation in Taiwanese KD patients. These observations provide insight into the association among inflammatory cytokines, matrix metalloproteinases, and CAL formation in KD. As a result, we may use MMP gene expression and DNA methylation as KD biomarkers to develop an effective KD diagnosis model.

This study has various limitations. First, we do

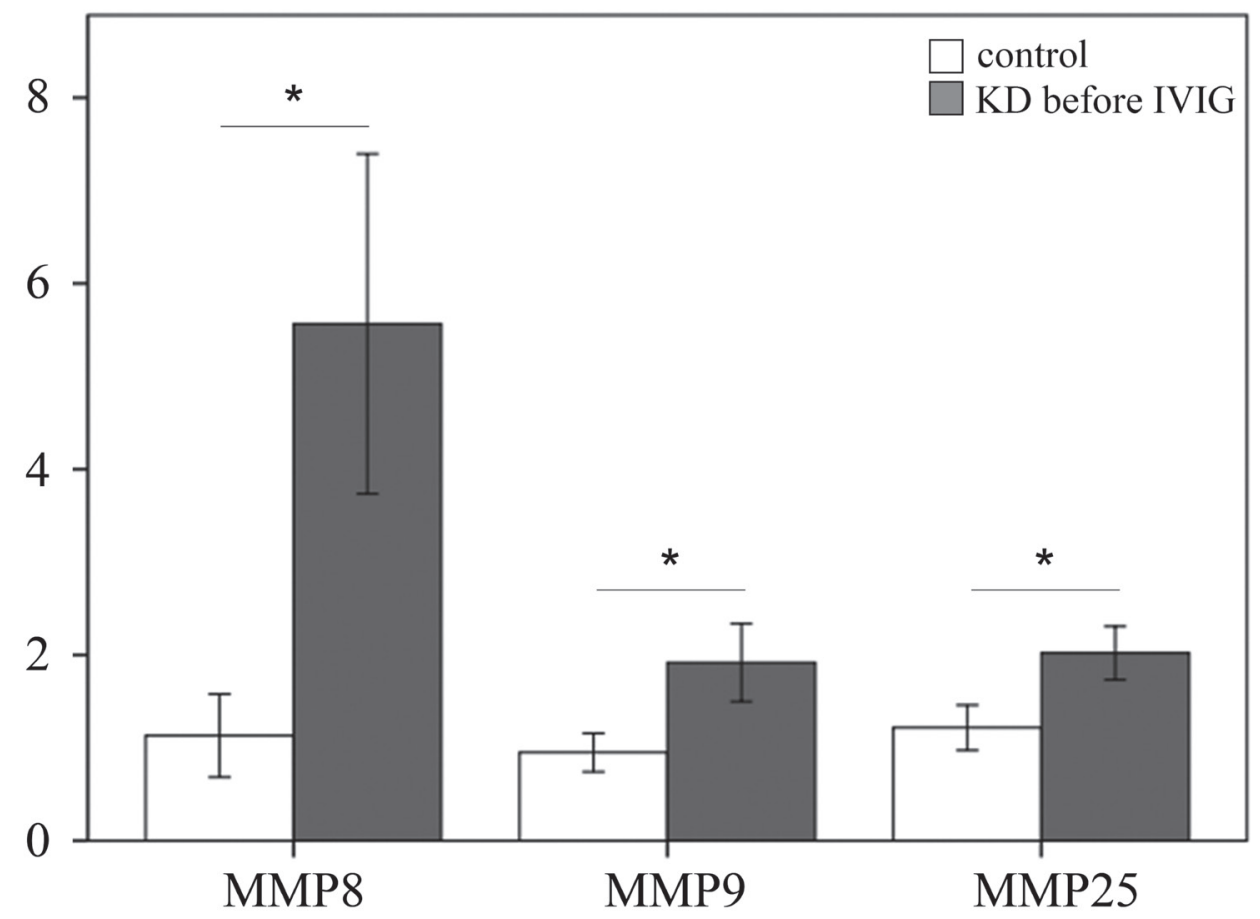

Figure 3: Analyses of matrix metalloproteinase (MMP) -8, -9, and -25 mRNA in the peripheral blood mononuclear cells of 31 patients with KD and 46 controls using a real-time quantitative polymerase chain reaction. Data are expressed as mean \pm standard error. *indicates a $\mathrm{p}<0.05$ between the groups. 
Table 4: Primers were designed to amplify target genes

\begin{tabular}{|c|c|c|c|c|c|}
\hline Gene symbol & Accession number & Hybridization & Primers (5' to 3') & \begin{tabular}{|l|} 
Primer \\
location
\end{tabular} & $\begin{array}{r}\text { Amplicon } \\
\text { size (bp) }\end{array}$ \\
\hline RNA18S5 & NR 003286.2 & forward & GTAACCCGTTGAACCCCATT & $1577-1597$ & \multirow{2}{*}{151} \\
\hline & & reverse & CCATCCAATCGGTAGTAGCG & $1708-1727$ & \\
\hline MMP8 & NM_002424 & forward & TTGGGTTGAATGTGACGG & $308-326$ & \multirow{2}{*}{156} \\
\hline & & reverse & GTATAGTTTCGAATCCTGTAGGT & $439-463$ & \\
\hline MMP9 & NM_004994 & forward & TCCAACCACCACCACAC & $1378-1396$ & \multirow{2}{*}{178} \\
\hline & & reverse & CGGACTCAAAGGCACAGTA & $1534-1555$ & \\
\hline MMP25 & NM_022468 & forward & GGACACTCACTTTGACGAT & $852-873$ & \multirow{2}{*}{192} \\
\hline & & reverse & GAGACAGGCGGTACTTG & $1026-1043$ & \\
\hline
\end{tabular}

not know if potential changes in methylation occur due to an association with decreased inflammation or due to the IVIG treatment. Second, we use total WBCs in this study, but determining whether specific types of blood cells, such as regulatory T cells, or specific markers show epigenetic changes with IVIG or KD would be productive and interesting.

\section{CONCLUSIONS}

Our report is the first to observe DNA hypomethylation, an increased MMP-9 transcript in KD compared to controls and an upregulation of MMP-9 in $\mathrm{KD}$ patients with CAL formation.

\section{MATERIALS AND METHODS}

\section{Patients}

For this study, we recruited a total of 54 subjects from Kaohsiung Chang Gung Memorial Children's Hospital in Taiwan in this study. Of those, 18 subjects were fever controls (with fever but not diagnosed to have KD), 18 subjects were healthy controls (with a history of neither fever nor KD), and the remaining 18 subjects were KD patients who had had samples collected both before and after IVIG treatment, met the American Heart

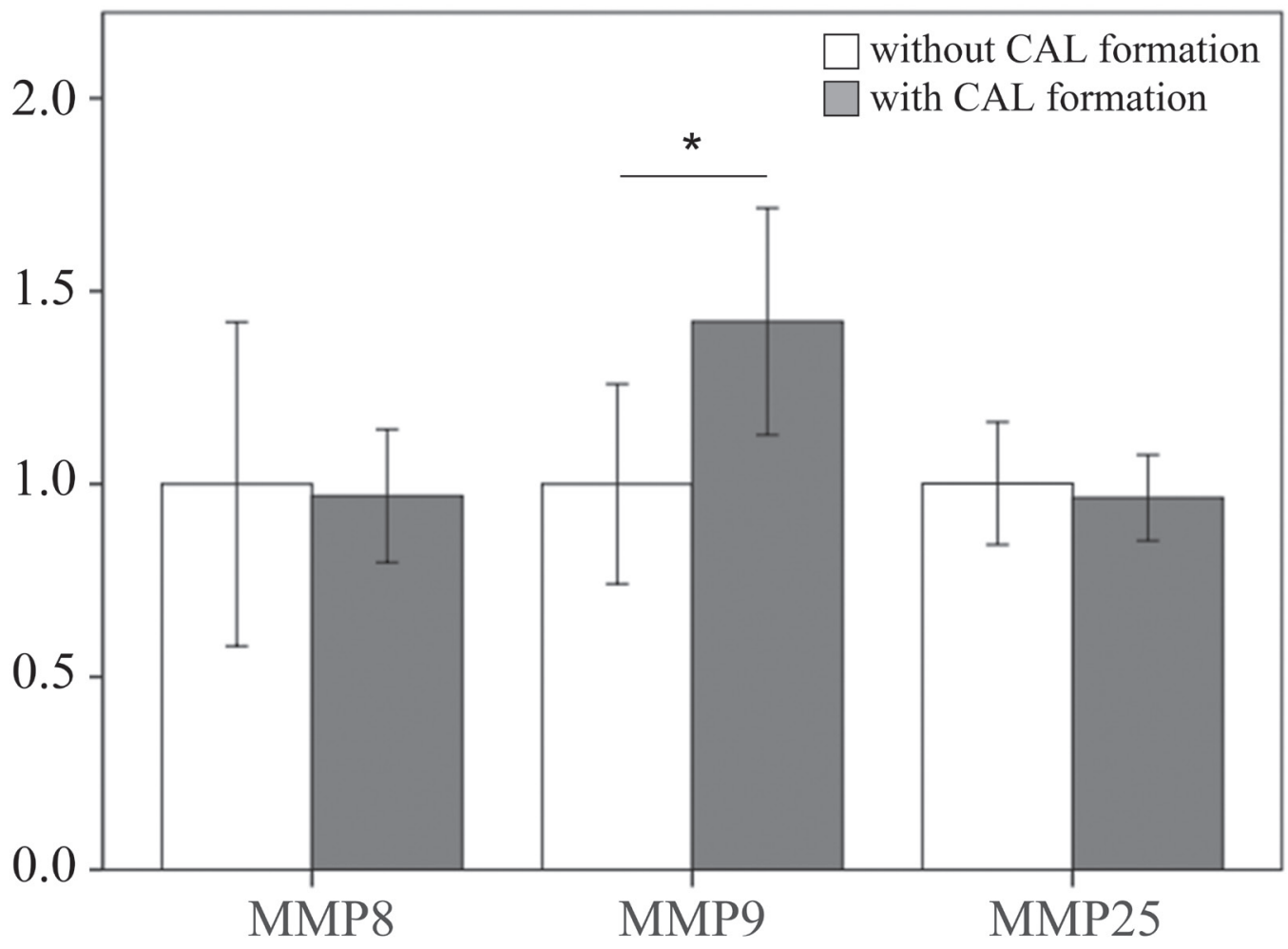

Figure 4: Comparison of matrix metalloproteinase 9 in patients with KD without $(n=22)$ and with $(n=9)$ coronary artery lesion before being treated with intravenous immunoglobulin. Data are presented as mean \pm standard error. $*$ indicates a $p<0.05$ between the groups. 
Association diagnosis criteria, which is characterized by prolonged fever for more than five days, conjunctivitis, diffuse mucosal inflammation, polymorphous skin rashes, indurative edema of the hands and feet associated with peeling of the finger tips, and non-suppurative lymphadenopathy $[39,40]$, and received IVIG treatment at the hospital. Using a separate cohort, we performed realtime quantitative PCR validations of mRNA levels in 31 KD patients, 23 healthy controls, and 23 febrile controls. The patients in the fever control group had diagnoses of acute tonsillitis, croup, acute bronchitis, or urinary tract infection. We took peripheral blood samples from KD patients at two points: prior to being treated with IVIG (pre-IVIG) and at least three weeks after completing IVIG treatment $(37.8 \pm 4.0$ days $)$, as described in our previous studies [36]. A CAL was defined as a coronary artery with an internal diameter of at least $3 \mathrm{~mm}$ (4 $\mathrm{mm}$ if the subject was older than 5 years) or a segment with an internal diameter at least 1.5 times larger than that of an adjacent segment, as observed through echocardiography $[14,41]$. This study received approval from the Chang Gung Memorial Hospital's Institutional Review Board (IRB No.:101-4618A3), and we obtained written informed consent from the parents or guardians of all subjects. All of the methods used comply with the relevant guidelines established.

\section{Experiment design}

First, we collected whole blood samples from the subjects and subjected said samples to WBC enrichment, as described in a previous study [12]. The enriched WBC samples were then subjected to either RNA or DNA extraction. We used an isolation kit (mirVana ${ }^{\mathrm{TM}}$ miRNA Isolation Kit, Catalog number: AM1560, Life Technologies, Carlsbad, CA) following the manufacturer's instructions to isolate total RNA and measured the collected RNA samples using Bioanalyzer (ABI) and Qubit (Thermo) to calculate both the quality (RIN value) and quantity of the RNA. All RNA samples passed the criterion of RIN $\geqq 7$. We isolated the DNA samples and treated them with bisulfite as previously described in another study [42].

\section{Gene expression profiling with microarray}

For strong, unbiased results, pooled RNA libraries were created by evenly pooling six RNA samples, which resulted in three pooled normal control, three fever control, three pre-IVIG, and three post-IVIG libraries. We submitted the pooled RNA samples to microarray assay to establish the gene expression profiles. We further performed profiling with GeneChip ${ }^{\circledR}$ Human Transcriptome Array 2.0 (HTA 2.0, Affymetrix, Santa Clara). We used the WT PLUS Reagent kit to prepare the RNA samples and then performed hybridization on the HTA 2.0 microarray chips. In accordance with the Affymetrix instruction manual, the raw data of the HTA 2.0 chips underwent quality control examination, as previously described [13].

\section{DNA methylation profiling with Illumina M450K BeadChip}

We used Illumina HumanMethylation450 (M450K) BeadChip to perform genome-wide screening of DNA methylation patterns. The M450K BeadChip program was created to detect methylation patterns of approximately $450,000 \mathrm{CpG}$ markers, thus spanning the entire human genome. Additional information about M450 BeadChip can be found at the following website: http://support.illumina.com/array/array_kits/ infinium_humanmethylation450_beadchip_kit.html.

For each M450K BeadChip assay, we applied $200 \mathrm{ng}$ of bisulfite-converted genomic DNA in accordance with the manufacturer's instructions, as previously described [12]. Then, we calculated the methylation percentage of cytosine for each $\mathrm{CpG}$ marker in each sample, referring to them as $\beta$ values.

\section{RNA isolation and real-time quantitative RT-PCR}

In order to quantify the mRNA levels of MMP8 , -9, and -25 , we performed real-time quantitative PCR using the ABI 7700 Sequence Detection System (TaqMan; Applied Biosystems, Inc., Foster City, CA). We separated the total mRNA from the WBC using an isolation kit (mirVana ${ }^{\mathrm{TM}}$ miRNA Isolation Kit, Catalog number: AM1560, Life Technologies, Carlsbad, CA), following the manufacturer's instructions. We performed PCR using a SYBR Green PCR Master Mix containing 10 $\mu \mathrm{M}$ of specific forward and reverse primers. The relative quantification of gene expression was carried out based on the comparative threshold cycle $\left(\mathrm{C}_{\mathrm{T}}\right)$ method, through which the target amount was determined to be $2^{-(\Delta \mathrm{CT}}$ target $-\Delta \mathrm{CT}$ calibrator) or $2^{-\Delta \Delta \mathrm{CT}}$ [43]. Primers were designed to amplify the target genes, which are provided in Table 4. We performed all experiments twice for verification and to validate the amplification efficiencies.

\section{Statistical analysis}

We have presented all data as mean \pm standard error. The chips that passed the quality control criteria were analyzed with Partek (Partek, St. Louis), commercial software designed specifically to analyze microarray data. Using Partek, we conducted ANOVA analysis and reported the $\mathrm{p}$-values of the comparisons of interest as previously described [13]. 
We applied Student's t-test or one-way ANOVA, as appropriate, to analyze the quantitative data. Furthermore, we evaluated any data changes before and after IVIG treatment with the paired sample $t$-test. All statistical analyses were carried out using SPSS version 22.0 for Windows XP (SPSS, Inc., Chicago, USA), and a twosided $\mathrm{p}$-value less than 0.05 was considered statistically significant.

\section{ACKNOWLEDGMENTS}

The authors would like to thank Xin-Yuan Cai for her assistance in this study.

\section{FUNDING}

This study received funding from the following grants: MOST: 105-2314-B-182-050-MY3 and MOST 103-2410-H-264-004 from the Ministry of Science and Technology of Taiwan and CMRPG8F1911, 1921, 1931, and 1941, and 8E0212 from Chang Gung Memorial Hospital in Taiwan. These institutes provided financial support but did not influence the way in which we collected, analyzed, or interpreted the data or wrote this manuscript.

\section{CONFLICTS OF INTEREST}

All of the authors hereby declare that they have no financial interests to disclose with regard to this article.

\section{REFERENCES}

1. Kawasaki T, Kosaki F, Okawa S, Shigematsu I, Yanagawa H. A new infantile acute febrile mucocutaneous lymph node syndrome (MLNS) prevailing in Japan. Pediatrics. 1974; 54: 271-6.

2. Newburger JW, Takahashi M, Burns JC, Beiser AS, Chung KJ, Duffy CE, Glode MP, Mason WH, Reddy V, Sanders SP, Shulman ST, Wiggins JW, Hicks RV, et al. The treatment of Kawasaki syndrome with intravenous gamma globulin. N Engl J Med. 1986; 315: 341-7. doi: 10.1056/ NEJM198608073150601.

3. Newburger JW, Takahashi M, Gerber MA, Gewitz MH, Tani LY, Burns JC, Shulman ST, Bolger AF, Ferrieri P, Baltimore RS, Wilson WR, Baddour LM, Levison ME, et al. Diagnosis, treatment, and long-term management of Kawasaki disease: a statement for health professionals from the Committee on Rheumatic Fever, Endocarditis, and Kawasaki Disease, Council on Cardiovascular Disease in the Young, American Heart Association. Pediatrics. 2004; 114: 1708-33.

4. Hopps E, Caimi G. Matrix metalloproteases as a pharmacological target in cardiovascular diseases. Eur Rev Med Pharmacol Sci. 2015; 19: 2583-9.
5. Korematsu S, Ohta Y, Tamai N, Takeguchi M, Goto C, Miyahara H, Kawano T, Izumi T. Cell distribution differences of matrix metalloproteinase-9 and tissue inhibitor of matrix metalloproteinase- 1 in patients with Kawasaki disease. Pediatr Infect Dis J. 2012; 31: 973-4. doi: 10.1097/INF.0b013e31825ba6b3.

6. Reindel R, Baker SC, Kim KY, Rowley CA, Shulman ST, Orenstein JM, Perlman EJ, Lingen MW, Rowley AH. Integrins alpha4 and alphaM, collagen1 A1, and matrix metalloproteinase 7 are upregulated in acute Kawasaki disease vasculopathy. Pediatr Res. 2013; 73: 332-6. doi: 10.1038/pr.2012.185.

7. Shimizu C, Matsubara T, Onouchi Y, Jain S, Sun S, Nievergelt CM, Shike H, Brophy VH, Takegawa T, Furukawa S, Akagi T, Newburger JW, Baker AL, et al. Matrix metalloproteinase haplotypes associated with coronary artery aneurysm formation in patients with Kawasaki disease. J Hum Genet. 2010; 55: 779-84. doi: 10.1038/jhg.2010.109.

8. Blankier S, McCrindle BW, Ito S, Yeung RS. The role of atorvastatin in regulating the immune response leading to vascular damage in a model of Kawasaki disease. Clin Exp Immunol. 2011; 164: 193-201. doi: 10.1111/j.13652249.2011.04331.x.

9. Wilson AS, Power BE, Molloy PL. DNA hypomethylation and human diseases. Biochim Biophys Acta. 2007; 1775: 138-62. doi: 10.1016/j.bbcan.2006.08.007.

10. Jeltsch A. Beyond Watson and Crick: DNA methylation and molecular enzymology of DNA methyltransferases. Chembiochem. 2002; 3: 274-93.

11. Li SC, Wang FS, Yang YL, Tiao MM, Chuang JH, Huang YH. Microarray Study of Pathway Analysis Expression Profile Associated with MicroRNA-29a with Regard to Murine Cholestatic Liver Injuries. Int J Mol Sci. 2016; 17. doi: 10.3390/ijms17030324.

12. Li SC, Chan WC, Huang YH, Guo MM, Yu HR, Huang FC, Kuo HC, Kuo HC. Major methylation alterations on the $\mathrm{CpG}$ markers of inflammatory immune associated genes after IVIG treatment in Kawasaki disease. BMC Med Genomics. 2016; 9 Suppl 1: 37. doi: 10.1186/s12920-0160197-2.

13. Huang YH, Li SC, Huang LH, Chen PC, Lin YY, Lin $\mathrm{CC}$, Kuo HC. Identifying genetic hypomethylation and upregulation of Toll-like receptors in Kawasaki disease. Oncotarget. 2017; 8: 11249-58. doi: 10.18632/ oncotarget.14497.

14. Kuo HC, Wang CL, Liang CD, Yu HR, Huang CF, Wang L, Hwang KP, Yang KD. Association of lower eosinophilrelated T helper 2 (Th2) cytokines with coronary artery lesions in Kawasaki disease. Pediatr Allergy Immunol. 2009; 20: 266-72.

15. Yu HR, Kuo HC, Sheen JM, Wang L, Lin IC, Wang CL, Yang KD. A unique plasma proteomic profiling with imbalanced fibrinogen cascade in patients with Kawasaki disease. Pediatr Allergy Immunol. 2009; 20: 699-707. 
16. Suzuki A, Kamiya T, Kuwahara N, Ono Y, Kohata T, Takahashi O, Kimura K, Takamiya M. Coronary arterial lesions of Kawasaki disease: cardiac catheterization findings of 1100 cases. Pediatr Cardiol. 1986; 7: 3-9.

17. Kuo HC, Wang CL, Liang CD, Yu HR, Chen HH, Wang L, Yang KD. Persistent monocytosis after intravenous immunoglobulin therapy correlated with the development of coronary artery lesions in patients with Kawasaki disease. J Microbiol Immunol Infect. 2007; 40: 395-400.

18. Kuo HC, Liang CD, Wang CL, Yu HR, Hwang KP, Yang KD. Serum Albumin Level Predicts Initial Intravenous Immunoglobulin Treatment Failure in Kawasaki Disease. Acta Paediatr. 2010.

19. Yamakawa R, Ishii M, Sugimura T, Akagi T, Eto G, Iemura M, Tsutsumi T, Kato H. Coronary endothelial dysfunction after Kawasaki disease: evaluation by intracoronary injection of acetylcholine. J Am Coll Cardiol. 1998; 31: 1074-80.

20. Mirhafez SR, Avan A, Tajfard M, Mohammadi S, Moohebati M, Fallah A, Ghazavi H, Savadi H, Ghayour Mobarhan M. Relationship between serum cytokines receptors and matrix metalloproteinase 9 levels and coronary artery disease. J Clin Lab Anal. 2016. doi: 10.1002/jcla.22100.

21. El-Aziz TA, Mohamed RH. Matrix metalloproteinase -9 polymorphism and outcome after acute myocardial infarction. Int J Cardiol. 2017; 227: 524-8. doi: 10.1016/j. ijcard.2016.10.109.

22. Klaus V, Tanios-Schmies F, Reeps C, Trenner M, Matevossian E, Eckstein HH, Pelisek J. Association of Matrix Metalloproteinase Levels with Collagen Degradation in the Context of Abdominal Aortic Aneurysm. Eur J Vasc Endovasc Surg. 2017. doi: 10.1016/j.ejvs.2016.12.030.

23. Newby AC. Metalloproteinase production from macrophages - a perfect storm leading to atherosclerotic plaque rupture and myocardial infarction. Exp Physiol. 2016; 101: 1327-37. doi: 10.1113/EP085567.

24. Ban JY, Kim SK, Kang SW, Yoon KL, Chung JH. Association between polymorphisms of matrix metalloproteinase 11 (MMP-11) and Kawasaki disease in the Korean population. Life Sci. 2010; 86: 756-9. doi: 10.1016/j.1fs.2010.03.012.

25. Sakata K, Hamaoka K, Ozawa S, Niboshi A, Yahata T, Fujii M, Hamaoka A, Toiyama K, Nishida M, Itoi T. Matrix metalloproteinase-9 in vascular lesions and endothelial regulation in Kawasaki disease. Circ J. 2010; 74: 1670-5.

26. Chen ZH, Wan GP, Gu XQ. [Effect of small interfering RNA on matrix metalloproteinase- 9 expression in vascular endothelial cells stimulated by serum from children with Kawasaki disease]. [Article in Chinese]. Zhonghua Xin Xue Guan Bing Za Zhi. 2009; 37: 837-40.

27. Lau AC, Duong TT, Ito S, Yeung RS. Matrix metalloproteinase 9 activity leads to elastin breakdown in an animal model of Kawasaki disease. Arthritis Rheum.
2008; 58: 854-63. doi: 10.1002/art.23225.

28. Lau AC, Duong TT, Ito S, Wilson GJ, Yeung RS. Inhibition of matrix metalloproteinase-9 activity improves coronary outcome in an animal model of Kawasaki disease. Clin Exp Immunol. 2009; 157: 300-9. doi: 10.1111/j.13652249.2009.03949.x.

29. Zhou C, Huang M, Xie L, Shen J, Xiao T, Wang R. IVIG inhibits TNF-alpha-induced MMP9 expression and activity in monocytes by suppressing NF-kappaB and P38 MAPK activation. Int J Clin Exp Pathol. 2015; 8: 15879-86.

30. Ling L, Ren M, Yang C, Lao G, Chen L, Luo H, Feng Z, Yan L. Role of site-specific DNA demethylation in TNFalpha-induced MMP9 expression in keratinocytes. J Mol Endocrinol. 2013; 50: 279-90. doi: 10.1530/JME-120172 .

31. Lu W, Li J, Ren M, Zeng Y, Zhu P, Lin L, Lin D, Hao S, Gao Q, Liang J, Yan L, Yang C. Role of the mevalonate pathway in specific $\mathrm{CpG}$ site demethylation on AGEsinduced MMP9 expression and activation in keratinocytes. Mol Cell Endocrinol. 2015; 411: 121-9. doi: 10.1016/j. mce.2015.04.019.

32. Chen KC, Wang YS, Hu CY, Chang WC, Liao YC, Dai CY, Juo SH. OxLDL up-regulates microRNA-29b, leading to epigenetic modifications of MMP-2/MMP-9 genes: a novel mechanism for cardiovascular diseases. FASEB J. 2011; 25: 1718-28. doi: 10.1096/fj.10-174904.

33. Soria-Valles C, Gutierrez-Fernandez A, Osorio FG, Carrero D, Ferrando AA, Colado E, Fernandez-Garcia MS, BonzonKulichenko E, Vazquez J, Fueyo A, Lopez-Otin C. MMP25 Metalloprotease Regulates Innate Immune Response through NF-kappaB Signaling. J Immunol. 2016; 197: 296302. doi: 10.4049/jimmunol.1600094.

34. Gomez I, Ozen G, Deschildre C, Amgoud Y, Boubaya L, Gorenne I, Benyahia C, Roger T, Leseche G, Galardon E, Topal G, Jacob MP, Longrois D, et al. Reverse Regulatory Pathway (H2S / PGE2 / MMP) in Human Aortic Aneurysm and Saphenous Vein Varicosity. PLoS One. 2016; 11: e0158421. doi: 10.1371/journal.pone.0158421.

35. Yen JH, Kocieda VP, Jing H, Ganea D. Prostaglandin E2 induces matrix metalloproteinase 9 expression in dendritic cells through two independent signaling pathways leading to activator protein 1 (AP-1) activation. J Biol Chem. 2011; 286: 38913-23. doi: 10.1074/jbc.M111.252932.

36. Kuo HC, Wang CL, Yang KD, Lo MH, Hsieh KS, Li SC, Huang YH. Plasma Prostaglandin E2 Levels Correlated with the Prevention of Intravenous Immunoglobulin Resistance and Coronary Artery Lesions Formation via CD40L in Kawasaki Disease. PLoS One. 2016; 11: e0161265. doi: 10.1371/journal.pone.0161265.

37. Liu R, Wu W, Chen L, Chen H, Zhang S. Transcriptional expression profiles of the main proteinases and their regulators in coronary artery ectasia patients' mononuclear cells. Acta Cardiol. 2016; 71: 157-63. doi: 10.2143/ AC.71.2.3141845. 
38. Huang YH, Hsu YW, Lu HF, Wong HS, Yu HR, Kuo HC, Huang FC, Chang WC, Kuo HC. Interferon-gamma Genetic Polymorphism and Expression in Kawasaki Disease. Medicine (Baltimore). 2016; 95: e3501. doi: 10.1097/ MD.0000000000003501.

39. Newburger JW, Takahashi M, Gerber MA, Gewitz MH, Tani LY, Burns JC, Shulman ST, Bolger AF, Ferrieri P, Baltimore RS, Wilson WR, Baddour LM, Levison ME, et al. Diagnosis, treatment, and long-term management of Kawasaki disease: a statement for health professionals from the Committee on Rheumatic Fever, Endocarditis and Kawasaki Disease, Council on Cardiovascular Disease in the Young, American Heart Association. Circulation. 2004; 110: 2747-71.

40. Kuo HC, Lo MH, Hsieh KS, Guo MM, Huang YH. HighDose Aspirin Is Associated with Anemia and Does Not Confer Benefit to Disease Outcomes in Kawasaki Disease. PLoS One. 2015; 10: e0144603. doi: 10.1371/journal. pone. 0144603 .
41. Kuo HC, Yang KD, Liang CD, Bong CN, Yu HR, Wang L, Wang CL. The relationship of eosinophilia to intravenous immunoglobulin treatment failure in Kawasaki disease. Pediatr Allergy Immunol. 2007; 18: 354-9.

42. Kuo HC, Chang JC, Yu HR, Wang CL, Lee CP, Huang $\mathrm{LT}$, Yang KD. Identification of an association between genomic hypomethylation of FCGR2A and susceptibility to Kawasaki disease and intravenous immunoglobulin resistance by DNA methylation array. Arthritis Rheumatol. 2015; 67: 828-36. doi: 10.1002/art.38976.

43. Yang YL, Wang FS, Li SC, Tiao MM, Huang YH. MicroRNA-29a Alleviates Bile Duct Ligation Exacerbation of Hepatic Fibrosis in Mice through Epigenetic Control of Methyltransferases. Int J Mol Sci. 2017; 18. doi: 10.3390/ ijms18010192. 